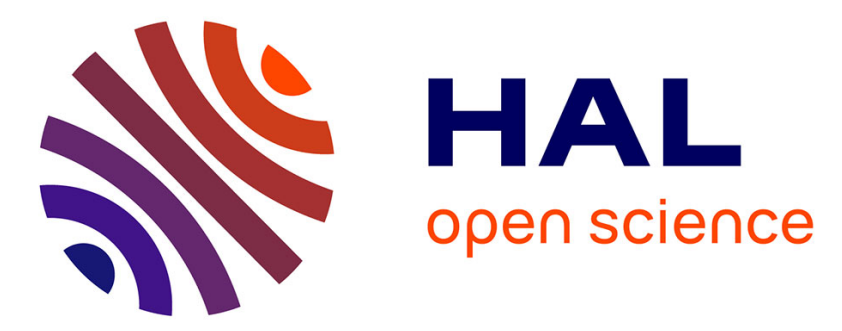

\title{
Une source de lumière pour le dosage isotopique de l'uranium par spectrométrie d'émission
}

\author{
Mathieu Ceccaldi, P. Goujon, J.P. Leicknam, G. Nief
}

\section{To cite this version:}

Mathieu Ceccaldi, P. Goujon, J.P. Leicknam, G. Nief. Une source de lumière pour le dosage isotopique de l'uranium par spectrométrie d'émission. J. Phys. Phys. Appl., 1963, 24 (S3), pp.65-68. 10.1051/jphysap:0196300240306500 . jpa-00212911

\section{HAL Id: jpa-00212911 https://hal.science/jpa-00212911}

Submitted on 1 Jan 1963

HAL is a multi-disciplinary open access archive for the deposit and dissemination of scientific research documents, whether they are published or not. The documents may come from teaching and research institutions in France or abroad, or from public or private research centers.
L'archive ouverte pluridisciplinaire HAL, est destinée au dépôt et à la diffusion de documents scientifiques de niveau recherche, publiés ou non, émanant des établissements d'enseignement et de recherche français ou étrangers, des laboratoires publics ou privés. 


\title{
UNE SOURCE DE LUMIERE POUR LE DOSAGE ISOTOPIQUE DE L'URANIUM PAR SPECTROMÉTRIE D'ÉMISSION
}

\author{
Par M. CECCALDI, P. GOUJON, J. P. LEICKNAM et G. NIEF (1), \\ Service des Isotopes stables. C. E. N., Saclay.
}

\begin{abstract}
Résumé. - On décrit un procédé de fabrication et d'excitation de lampès sans électrodes au tétrachlorure ou au tétraiodure d'uranium qui émettent le spectre optique de l'uranium avec une intensité, une pureté et une stabilité suffisantes pour permettre l'analyse isotopique avec le spectromètre interférentiel " Hypéac ». Le procédé de fabrication est simple et rapide et peut être utilisé pour des analyses de routine.
\end{abstract}

\begin{abstract}
A process is described for producing and exciting electrodeless uranium tetrachloride or tetraiodide lamps emitting the optical spectrum of uranium with an intensity, a purity, and a stability sufficient to allow the isotopic analysis to be carried out with the "Hypéac " interference spectrometer. The process of fabrication is simple and rapid, and can be used for routine analyses.
\end{abstract}

I. Introduction. - La possibilité théorique du dosage isotopique de l'uranium par spectrométrie d'émission a été démontrée [1] en particulier par les travaux du $\mathrm{P}^{\mathrm{r}}$ Jacquinot du Laboratoire Aimé Cotton de Bellevue et de Gerstenkorn du Laboratoire de Chimie du C. E. A. [2]. Nous nous sommes posés le problème d'étudier une source de lumière de fabrication aisée, émettant le spectre de l'uranium de façon suffisamment stable et intense:

Notre choix s'est porté sur les tubes à décharge haute fréquence sans électrodes, qui possèdent en général les caractéristiques requises d'intensité et de stabilité. Le défaut, dont sont parfois taxées les lampes de ce type, d'élargir les raies, paraît $a$ priori être jugé peu gênant, ce qui a été confirmé expérimentalement par suite de la grandeur du déplacement isotopique du spectre de l'uranium.

Le spectre d'émission de l'uranium est dû à la recombinaison électronique des ions $\mathrm{U}^{+}$. Il est connu que les sels $\mathrm{UX}_{4}$ (X pourrait être $\mathrm{Cl}, \mathrm{Br}$ ou I) conviennent pour produire ce spectre $\left({ }^{2}\right)$. La pratique habituelle [3], [4], [5], [6], [7] consiste à placer une quantité de l'ordre du milligramme de sel d'uranium dans un tube en quartz dans lequel règne une pression de quelques millimètres d'un gaz rare, argon ou néon, et d'exciter cette lampe en ondes décimétriques avec une puissance de quelques dizaines de watts [3], [4], [5], [6]. Nous avons été conduits, pour des raisons pratiques, à modifier sensiblement ces conditions. En effet, les tétrachlorure et tétraiodure d'uranium sont très sensibles aux traces d'oxygène, solvants chlorés et vapeur d'eau.

(1) Commissariat à l'Énergie Atomique. C. E. N. S., Saclay. Service des Isotopes Stables. Section de Spectrométrie de Masse. B. P. no 2, Gif-sur-Yvette (Seine-etOise).

(2) Quelques tentatives pour obtenir le spectre de l'uranium par décharge dans $\mathrm{UF}_{6}$ n'ont pas abouti.
Pour faire une lampe qui fonctionne avec seulement $1 \mathrm{mg}$ de $\mathrm{UX}_{4}$, il est nécessaire de procéder à une purification soignée des réactifs et à un dégazage très poussé de l'ampoule de la lampe. La fabrication de ces lampes est dans ces conditions une opération délicate et surtout très longue, peu compatible avec l'exécution d'analyses de routine. En utilisant des quantités de $\mathrm{UCl}_{4}$ plus importantes, $30 \mathrm{mg}$, ces conditions de propreté sont beaucoup moins strictes, et il est possible de fabriquer une lampe fonctionnant correctement en moins de 2 heures. L'excitation de ces lampes en ondes décimétriques n'est plus possible.

En effet, les pertes diélectriques dans le tétrahalogénure d'uranium en excès et ses produits de décomposition portent la lampe à une température élevée. La pression devient trop grande, la décharge instable, le spectre continu intense. En abaissant la fréquence d'excitation de quelques milliers de mégacycles à quelques dizaines de mégacycles, ces pertes disparaissent et on obtient un fonctionnement parfaitement stable de ces lampes pendant plusieurs heures, temps très largement suffisant pour faire une analyse.

II. Fabrication des lampes. - 1. Préparation DU TÉtrahalogénuRe D'URANium $\left(^{3}\right)$. - a) Tétrachlorure d'uranium. - $\mathrm{UCl}_{4}$ est produit par réaction de $\mathrm{CCl}_{4}$ sur un oxyde d'uranium en ampoule scellée portée à $450^{\circ}$ pendant 15 minutes [8], [9], [10], [11], [12], [13]. Le composé d'uranium à analyser est transformé en nitrate d'uranyle qui est transformé en oxyde par calcination à l'air à $800{ }^{\circ} \mathrm{C}$.

$200 \mathrm{mg}$ d'oxyde et $5 \mathrm{~cm}^{3}$ de tétrachlorure de car-

(3) Les résultats comparables obtenus avec le tétrachlorure et le tétraiodure d'uranium ne nous ont pas incités à entreprendre la préparation de $\mathrm{UBr}_{4}$. 
bone sont introduits dans une ampoule de pyrex de $150 \mathrm{ml}$. Un pompage partiel sur cette ampoule amène le tétrachlorure de carbone à ébullition, ce qui chasse les gaz étrangers de l'ampoule. Cette opération est stoppée quand la moitié du tétrachlorure a été évacuée. L'ampoule est alors scellée, placée dans un cylindre métallique de protection et l'ensemble placé dans un four où il est chauffé à $450^{\circ} \mathrm{C}$ pendant 15 minutes. Après refroidissement, l'ampoule est ouverte sous hotte (la réaction produit du phosgène) ${ }^{\bullet}$ le tétrachlorure d'uranium rincé plusieurs fois au $\mathrm{CCl}_{4}$, séché et conservé sous vide ou sous azote sec.

b) Tétraiodure d'uranium. - On utilise l'action de l'iodure d'aluminium sur un oxyde d'uranium [5]. Comme précédemment, les produits sont portés à $450^{\circ} \mathrm{C}$ pendant $15 \mathrm{mn}$ dans une ampoule en verre pyrex scellée après qu'un vide partiel y ait été fait. Il est possible de préparer l'iodure d'aluminium in situ, en introduisant dans l'ampoule l'oxyde d'uranium, l'aluminium et de l'iode en excès. Après réaction dans le four, l'excès d'iode est éliminé par pompage. La séparation de $\mathrm{UI}_{4}$ de l'alumine et de la petite quantité d'oxyde d'uranium non transformé se fera par sublimation sous vide au cours de la préparation des lampes.

2. Remplissage Des lampes. - Les lampes sans électrode sont préparées à partir de tubes de quartz de diamètre intérieur 6 à $8 \mathrm{~mm}$, auxquels on a donné la forme schématisée par la figure 1. La partie A (longueur $10 \mathrm{~cm}$ environ) permet la sublimation de l'halogénure d'uranium ; la partie B (40 à $60 \mathrm{~mm}$ de longueur) constituera le corps de la source ; la partie $\mathrm{C}$ sert de réceptacle provisoire pendant la première partie du dégazage; enfin l'extrémité D sera raccordée à la ligne à vide.

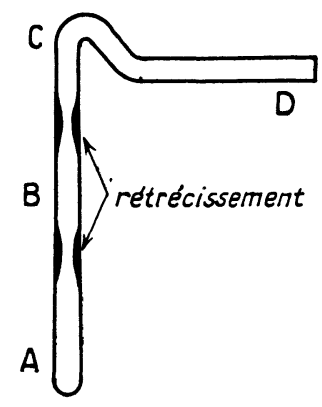

FIg. 1.

Mode opératoire. - L'introduction du tétrahalogénure d'uranium dans cette lampe se fait dans un caisson étanche à l'abri de l'humidité de l'air. La prise de départ est d'environ $50 \mathrm{mg}$. On bouche ( D ) provisoirement pendant le transfert sur la ligne à vide; on intercale un piège à azote liquide pour éviter la pollution de la ligne. Le vide $\left(10^{-3}\right.$ au minimum) doit rester stable pendant un quart

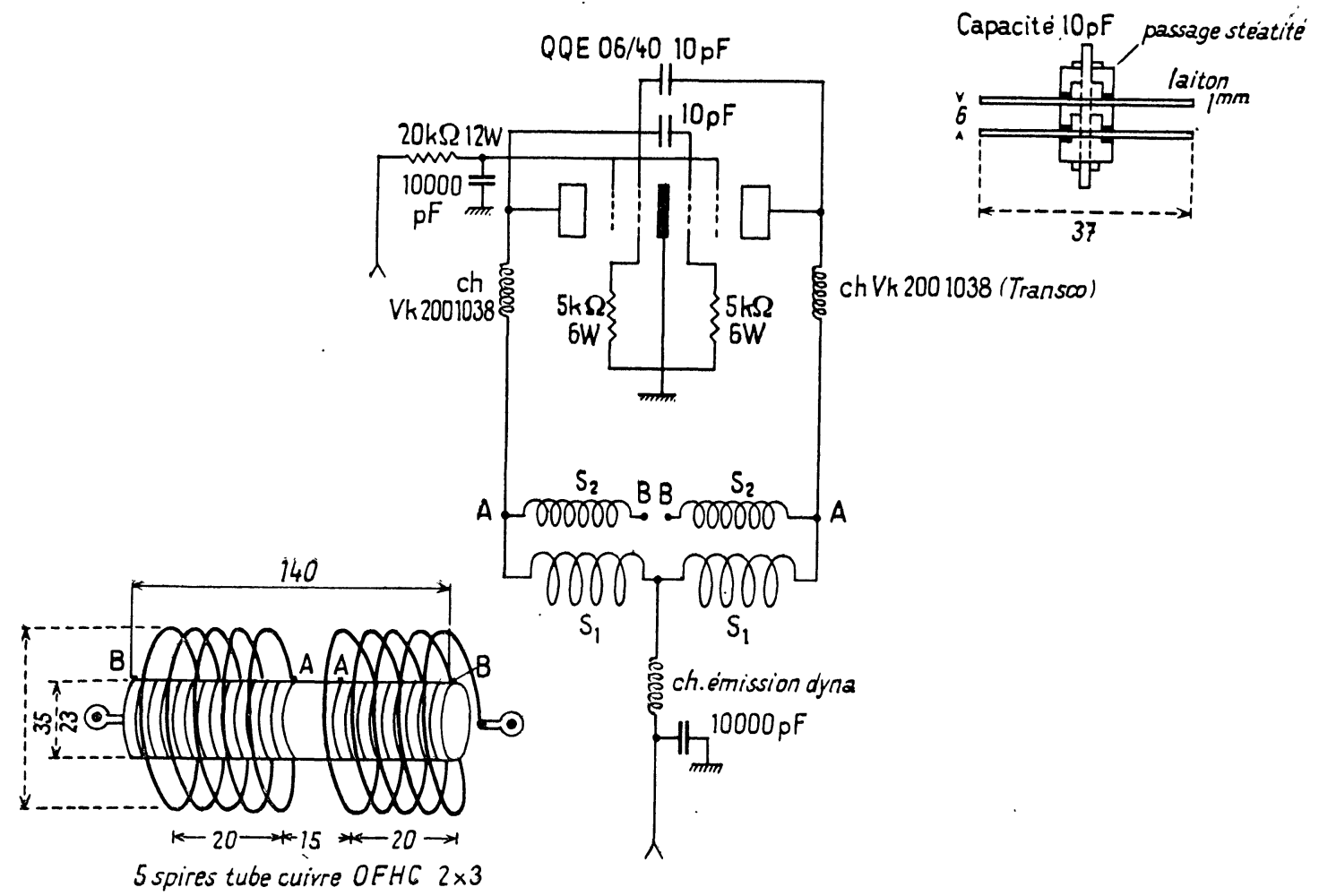

FIG. 2. 
d'heure environ. On place la partie A vers le haut, l'halogénure venant se rassembler en $\mathrm{C}$. On procède alors au dégazage de la partie $\mathrm{A}$ avec un petit four électrique porté à 1100 à $1200{ }^{\circ} \mathrm{C}$ pendant quelques minutes. On enlève le four et après complet refroidissement, on retourne la lampe de façon à rassembler le composé dans le fond de $\mathrm{A}$. On procède alors au dégazage de la partie $\mathrm{B}$, à la même température pendant une dizaine de minutes. Après complet refroidissement, on sublime le produit par chauffage de la partie A, en laissant en communication avec la ligne à vide, de façon à éliminer les produits plus volatils que $\mathrm{UCl}_{4}$ ou $\mathrm{UI}_{4}$. On sublime environ la moitié de la prise de départ. On soude alors au niveau de l'étranglement séparant $\mathrm{A}$ de $\mathrm{B}$, et on élimine la partie A. On introduit un gaz rare (argon, néon, ...) à une pression de 0,8 à $2 \mathrm{~mm}$ de mercure. Le corps de la lampe B est séparé après soudure au niveau du rétrécissement supérieur.

Remarque : Obtention des sources-étalons. - On utilise des oxydes de composition isotopique déterminée par spectrométrie de masse. A partir de quelques échantillons d'oxydes étalons, il est possible de réaliser une série d'étalons secondaires par pesées et mélanges, l'homogénéité du mélange étant obtenue par dissolution nitrique et calcination.

III. Excitateur. - Nous avons effectué alors des essais avec des générateurs d'onde métrique de puissances diverses, qui ont montré : que la lampe pouvait fonctionner à des températures très basses $\left(250^{\circ} \mathrm{C}\right)$; qu'un champ élevé est nécessaire sans

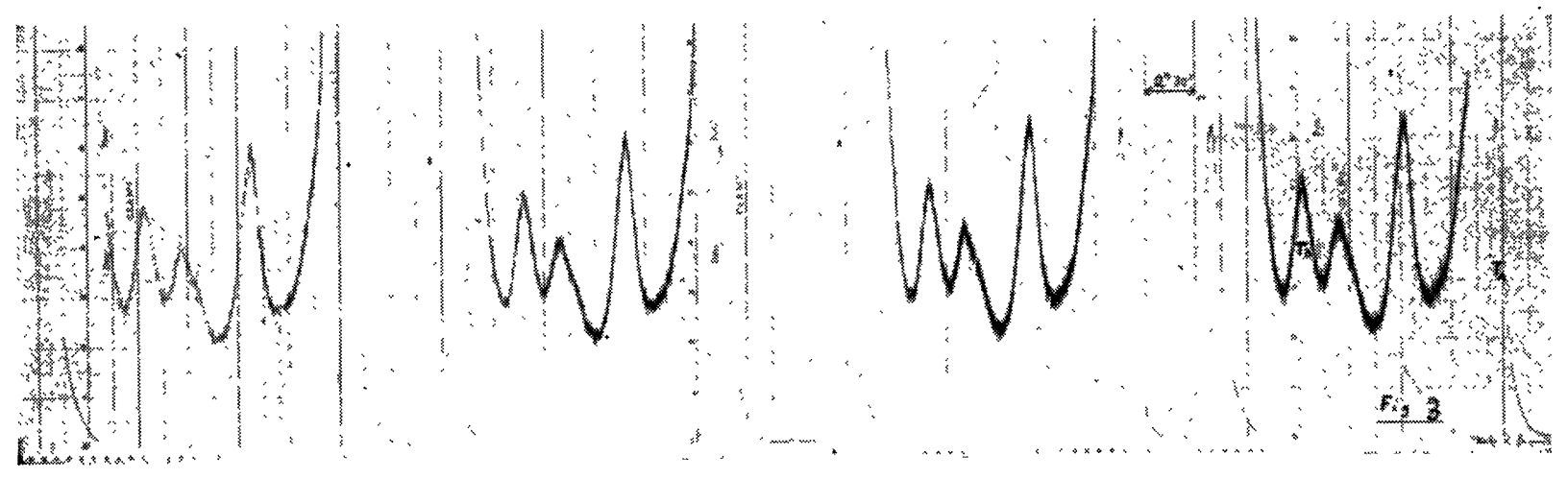

FIG. 3.

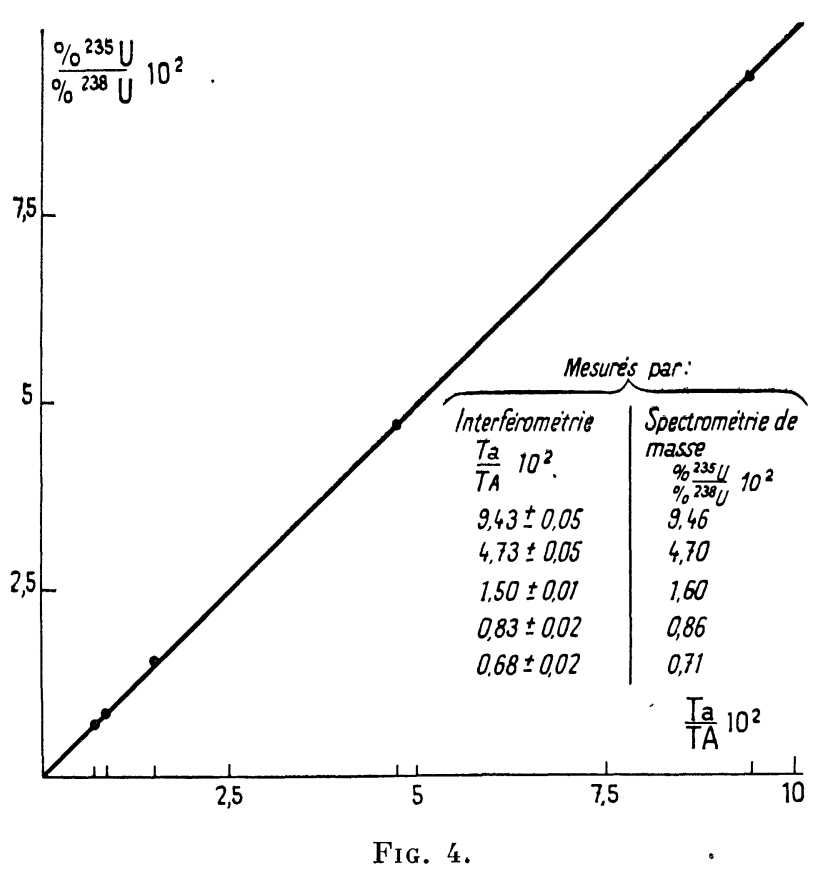

qu'il soit besoin de disposer d'une grande puissance.

Ces conditions nous ont conduits au montage de la figure 2. C'est un oscillateur symétrique à couplage capacitif.

Le montage doit être entouré d'un blindage suffisamment efficace pour que le détecteur (photomultiplicateur et amplificateur à courant continu de $10^{8} \Omega$ de résistance d'entrée) ne soit pas perturbé par le rayonnement de l'oscillateur. La lampe choisie est une double tétrode d'émission petite puissance (QQE 06/40).

La self du circuit oscillant est réalisée par $2 \times 5$ spires de tube de laiton électrolytique $($ OFMC $2 \times 3)$; elle est fixée sur des colonnettes de stéatite. Dans l'axe, on centre un tube de silice sur lequel est bobiné le secondaire du transformateur. Ce secondaire est constitué par $2 \times 18$ spires de fil de cuivre émaillé ( $\varnothing=1 \mathrm{~mm})$ maintenus régulièrement espacés (intervalles de $1 \mathrm{~mm}$ ); on ménage au milieu un espace libre de $15 \mathrm{~mm}$ pour le passage de la lumière.

Les selfs de choc des circuits plaques évitent les accrochages parasites qui se produisent lorsque la 
température des lampes croît trop. La haute tension est actuellement fournie par trois chassis d'alimentation stabilisée standard de 250 volts (type TALS) montés en série. Les lampes placées dàns ces conditions s'allument facilement; elles prennent leur régime d'équilibre en un quart d'heure environ.

IV. Résultats. - a) Brillance. - La brillance de ces lampes est au moins égale à celle des lampes à cathode creuse en uranium massif. Elle est suffisante pour donner un signal facilement mesurable pour ${ }^{235} \mathrm{U}$ dans de l'uranium de composition isotopique naturelle.

b) Stabilité. - Après un temps de mise en route d'environ 15 minutes, les signaux présentent une dérive régulière d'environ $0,3 \%$ par minute. Cette dérive étant régulière n'affecte pas la précision des mesures (fig. 3). c) Étalonnage. - Nous avons fabriqué, à titre d'exemple, un certain nombre de lampes avec de l'uranium de composition isotopique variant de $0,7 \%$ à $10 \%$ connue par spectrométrie de masse. La figure $\mathrm{n}^{0} 4$ donne le rapport des signaux 235 et 238 (Ta et TA) en fonction des rapports ${ }^{235} \mathrm{U} /{ }^{238} \mathrm{U}$ mesurés par spectrométrie de masse.

Note. - Par suite des irrégularités de fabrication des sources, la puissance nécessaire pour obtenir un bon fonctionnement varie d'une lampe à l'autre. Nous avons légèrement modifié le montage initial de l'excitateur : la variation de puissance est obtenue en agissant sur la tension de grille-écran. Pour cela on change la valeur de la résistance de cette grille grâce à un jeu de résistances en série, montées sur un contacteur. La puissance peut ainsi passer de $40 \mathrm{~W}$ à $100 \mathrm{~W}$ environ.

Manuscrit reçu le 15 septembre 1962.

\section{BIBLIOGRAPHIE}

[1] Rотн (E.), Nouveau traité de Chimie Minérale (publié sous la direction du Pr P. Pascal), tome XV, 1 er fascicule, p. 38 .

[2] Gerstenkorn (S.), Rapport C. E. A., no 845.

[3] Meggers (W. F.) et Westfall (F. O.), J. Res. Nat. Bur. Stand., 1950, 44, 447.

[4] Forrester (A. T.), Gadmindsen (R. A.) et Johnson (P. O.), J. Opt. Soc. Amer., 1956, 46, 339.

[5] Tomkins (F. S.) et Fred (M.), J. Opt. Soc. Amer., 1957, 47, 1087.

[6] Bovey (L.) et Wyse (H.), A. E. R. E., R. 2976.
[7] Johannin (A.), Fabrication de lampes sans électrode (travail non publié).

[8] Watts (C. W.) et Bell (C. A.), J. Chem. Soc., 1878, 33,442 .

[9] Meyer (L.), Ber., 1887, 20, 681.

[10] Colani (A.), Ann. Chim. et physique, 1907, 12 (8), 66-80.

[11] Cambonlives (P.), C. R. Acad. Sci., Fr., 1910, 150, 175.

[12] Michael (A.) et Murphy (A. Jr.), Amer. Chem. J., 1910, 44, 365-384.

[13] Steele (G.), Report RL-4, 1943, 6, 113, April 13. 